\title{
A community-led initiative for training in reproducible research
}

Susann Auer $\mathrm{PhD}^{1^{*}}$, Nele A. Haelterman $\mathrm{PhD}^{2^{*}}$, Tracey L. Weissgerber $\mathrm{PhD}^{3}$, Jeffrey C. Erlich $\mathrm{PhD}^{4}$, Damar Susilaradeya $\mathrm{PhD}^{5}$, Magdalena Julkowska $\mathrm{PhD}^{6}$, Małgorzata Anna Gazda $\mathrm{PhD}^{7,8}$, Reproducibility for Everyone Team, Benjamin Schwessinger PhD ${ }^{9 \#}$, Nafisa M. Jadavji PhD ${ }^{10 \#}$

Reproducibility for Everyone team: Angela Abitua, Anzela Niraulu, Aparna Shah, April Clyburne-Sherin, Benoit Guiquel, Bradly Alicea, Caroline LaManna, Diep Ganguly, Eric Perkins, Helena Jambor, Ian Man Ho Li, Jennifer Tsang, Joanne Kamens, Lenny Teytelman, Mariella Paul, Michelle Cronin, Nicolas Schmelling, Peter Crisp, Rintu Kutum, Santosh Phuyal, Sarvenaz Sarabipour, Sonali Roy, Susanna M Bachle, Tuan Tran, Tyler Ford, Vicky Steeves, Vinodh Ilangovan, Ana Baburamani, Chris Gahstrom, Susanna Bachle,

*These authors contributed equally to this work

${ }^{\#}$ Corresponding authors

${ }^{1}$ Department of Plant Physiology, Institute of Botany, Faculty of Biology, TU Dresden, Germany

${ }^{2}$ Department of Molecular and Human Genetics, Baylor College of Medicine, Houston, USA

${ }^{3}$ Berlin Institute of Health at Charité - Universitätsmedizin Berlin, QUEST Center, Charitéplatz 1, 10117 Berlin, Germany

${ }^{4}$ NYU-ECNU Institute of Brain and Cognitive Science at NYU Shanghai; Shanghai Key Laboratory of Brain Functional Genomics (Ministry of Education), East China Normal University, Shanghai, China

${ }^{5}$ Medical Technology Cluster, Indonesian Medical Education and Research Institute (IMERI), Faculty of Medicine Universitas Indonesia, Jakarta, Indonesia

${ }^{6}$ Boyce Thompson Institute, Ithaca, NY, USA

${ }^{7}$ CIBO/InBIOO, Centro de Investigação em Biodiversidade e Recursos Genéticos, Campus Agrário de Vairão, Universidade do Portio, 4485-661 Vairão, Portugal

${ }^{8}$ Departamento de Biologia, Faculdade de Ciências, Universidade do Portio, Porto, Portugal

${ }^{9}$ Research School of Biology, Australian National University, Canberra, ACT, Australia, benjamin.schwessinger@anu.edu.au

${ }^{10}$ Department of Biomedical Science, Midwestern University; Department of Neuroscience, Carleton University, njadav@ midwestern.edu 


\section{Abstract}

Open and reproducible research practices increase the reusability and impact of scientific 3 research. Factors affecting the reproducibility of results can arise from nearly every aspect of the scientific process and most can be overcome by improved education in roducible research practices. We present the Reproducibility for initiative that aims to provide training via series of customizable workshop modules that 57 covers the conceptual framework of reproducible research practices, followed by an 58 overview of actionable research practices. Our workshops target researchers at all levels 59 and across disciplines. To date, the R4E initiative has reached over two-thousand 60 researchers worldwide. R4E is volunteer-led and demonstrates how a shared set of 61 materials form the basis for a global initiative to improve reproducibility in science. All 62 workshop materials, including accompanying resources, are available under a CC-BY 4.0 63 license at www.repro4everyone.org.

64

65

66 Keywords: rigor, reproducibility, transparency, FAIR data, accessible protocols, 67 68 transparent data processing, workshop, training, open science

Abbreviations: Electronic lab notebooks, ELN; Findable Accessible Interpretable 71 


\section{Why is training in reproducibility needed?}

Reproducibility represents the ability to regenerate a result using the same dataset and data analysis workflow, while replicability reflects the ability to obtain similar results in a different experimental system (Leek et al., 2015; Schloss, 2018). Here, we use the term reproducibility to capture both these concepts at once as is often done in the life sciences (Barba, 2018). Since scientific progress is enabled by building on each other's observations and results, reproducibility is a critical element of science. Despite its importance for progress, studies have shown that it can be quite challenging to reproduce peer-reviewed results (Baker and Penny, 2016; Freedman et al., 2015). In the past few years, several multi-center projects have assessed the reproducibility rate in various scientific fields and have identified major factors that are critical for repeating and confirming scientific results (Alsheikh-Ali et al., 2011; Amaral et al., 2019; Baker et al., 2014; Button et al., 2013; Collaboration, 2015; Cova et al., 2020; Friedl, 2019; Hardwicke et al., 2018; Lazic, 2010; Marqués et al., 2020; Shen et al., 2012; Stevens, 2017; Strasak et al., 2007; Weissgerber et al., 2019, 2015).

The factors that control the reproducibility of an experiment can be grouped into four categories shown in Figure 1. The first represents technical factors, such as variability in reagents or materials used to perform research. The second category contains factors related to flaws in study design and/or statistical analysis such as the use of inappropriate controls, insufficient sample sizes to properly power the study, inappropriate statistical analyses, underpowered studies, and others. The third category contains human factors, which includes insufficient description of methods and the use of reagents or organisms that are not shared. In addition, scientific misconduct, such as hypothesizing after results have been obtained (HARKing) (Kerr, 1998) or P-hacking (Fraser et al., 2018; Head et al., 2015; Miyakawa, 2020) are hard to detect and contribute to confirmation and publication bias issues. Lastly, external factors that are beyond the researchers' control negatively impact, these can include scientific rewards such as a high impact publication and paywalls that restrict access to crucial information. Going forward, developing solutions to minimize these confounding factors will be of vital importance to improve scientific integrity and to further accelerate the advancement of the scientific enterprise (Botvinik-Nezer et al., 2020; Fomel and Claerbout, 2009; Friedl, 2019; Gentleman and Temple Lang, 2007; Mangul et al., 2019; Mesirov, 2010; Peng, 2011; "Rigor and Reproducibility | National Institutes of Health (NIH)," 2020). 


\begin{tabular}{|c|c|}
\hline $\begin{array}{l}\text { Technical } \\
\text { - Contaminated cell lines } \\
\text { - Unvalidated antibodies } \\
\text { and kits } \\
\text { - Natural variability } \\
\text { - Batch effects }\end{array}$ & $\begin{array}{l}\text { Study Design \& Statistics } \\
\text { : Design flaws } \\
\text { : Misunderstanding statistics } \\
\text { : } \text { P-haRking } \\
\text { - Confirmation bias } \\
\text { - Selective reporting }\end{array}$ \\
\hline $\begin{array}{l}\text { Human } \\
\text { - Insufficient detail about methods, } \\
\text { materials } \\
\text { - Poor sharing of reagents, data, } \\
\text { and code } \\
\text { - Lack of version control } \\
\text { - Mistakes } \\
\text { - Publication bias }\end{array}$ & $\begin{array}{l}\text { External } \\
\text { - Rewards for significant results, } \\
\text { publication in high impact journals } \\
\text { - Lack of incentives for responsible } \\
\text { research practices } \\
\text { - Paywalls } \\
\text { - Hyper competitiveness } \\
\text { - Fraud } \\
\text { - Sensitivity to conditions, equipment, } \\
\text { and staff }\end{array}$ \\
\hline
\end{tabular}

Figure 1. Approximation of the classification of categories that contribute to irreproducible scientific results. The factors include technical, human, errors in study design and statistical analysis, and external. Specific examples have been listed under each factor that contribute to irreproducible scientific results.

While the problems with experimental reproducibility have been known for decades, we have only recently seen a growing interest in reproducibility-promoting methods and technologies among the scientific and academic community, as well as among the general public (Begley and Ellis, 2012; Munafò et al., 2017; Prinz et al., 2011). Within the scientific community, systemic solutions and tools are being developed that allow scientists to efficiently share research materials, protocols, data, and computational analysis pipelines (some of these tools are covered in our training materials, see Box 1). Despite their transformative potential, these tools are underutilized, as most researchers are unaware of their existence, or do not know how to incorporate them in their daily workflows. Integrating these tools into the standard scientific workflow has the potential to shift the scientific community towards a more transparent and reproducible future. Educational initiatives with open-source materials can significantly increase the reach of teaching materials (Lawrence et al., 2015), to accelerate the uptake of best practices and existing tools for reproducible research. Several initiatives exist that offer tutorials or seminars on some aspects of reproducibility (Box 2). While they each have their strengths, none of them individually offer a scalable solution to the existing training gap in reproducibility. Our trainings are scalable. From a dozen attendees in an intensive workshop to a few hundred participants in an introductory workshop that can attend at once in a virtual format or a large venue. However, the reproducibility movement worldwide is growing, and as different initiatives cover various aspects of the training process, they can together help bridge the reproducible training gap.

\section{Box 1: Unit Topics}

The units included in the standard introductory workshop cover a range of skills and tools needed to conduct reproducible research. Below are examples of content that has been used in previous workshops. The specific content of each workshop can vary and 
is adjusted to the audience and event.

1. The reproducibility framework: Reproducible research practices allow others to repeat analyses and corroborate results from a previous study. This is only possible when authors have provided all necessary data, methods and computer codes (Figure 2). Our reproducibility toolbox includes reproducible practices for organization, documentation, analysis, and dissemination of scientific research.

2. Organization, data management and file naming: An effective data management plan, including clear file naming conventions, prevents problems such as lost data, difficulties identifying the most recent version of a file, the inability to locate files after team members leave the laboratory, or difficulties in finding or interpreting files years after the project is completed. This section describes techniques to ensure that all project files are easy to identify and locate and that they are appropriately documented.

3. Electronic lab notebooks: Electronic lab notebooks (ELNs) overcome many of the limitations of paper lab notebooks - they are searchable, cannot be damaged or misplaced, and are easy to back-up and share with collaborators. This section discusses available electronic lab notebooks and strategies for selecting the electronic lab notebook that meets the needs of an individual research team.

4. Preregistrations and protocol sharing: Scientific publications often lack essential details needed to reproduce the methods described. Preregistrations of planned research include details of the methods and tools that will be used in the project and provide transparency of the intended analyses and outcome. Protocol repositories allow researchers to share detailed, step-by-step protocols, which can be cited in scientific papers. Repositories also make it easy to track changes in protocols over time by incorporating version control, allowing researchers to post updated versions of protocols from their own lab, or adapted versions of protocols that were originally shared by other research groups. This section describes strategies for creating effective "living protocols" that other research teams can easily locate, follow, cite and adapt.

5. Biological material and reagent sharing: Laboratories regularly produce specialized materials and organisms, such as reagents, plasmids, seeds and organism strains. Access to these materials is essential to reproduce and expand upon published work. Repositories maintain reagents and biological materials deposited by scientists, and also make these materials accessible to the scientific community for a small or symbolic donation. Nonetheless, many laboratories do not use repository resources to share their materials, and thus limit their outreach and impact. This section introduces the concept of material repositories, which allow investigators access to materials without investing time and resources to recreate, maintain, verify and distribute their own or another researcher's reagents.

6. Data visualization and figure design: Figures show the key findings of a study and should allow readers to critically evaluate the data and structures behind them. 
As an example, scientists routinely use the default plots of spreadsheet software such as bar graphs for presenting continuous data (Weissgerber et al., 2015). This is a problem, as many data distributions can lead to the same bar or line graph and the actual data may suggest different conclusions from the summary statistics alone. This section illustrates strategies for replacing bar graphs with more informative alternatives (i.e. dot, box, or violin plots), provides guidance on choosing the visualization best suited for various data structures and images, and provides a brief overview of tools for creating more effective, appealing and informative graphics and figures.

7. Bioinformatic tools: The sample size and number of data points (in multidimensional data) in research studies has greatly increased in the last decade. Bioinformatic tools for analyzing large data sets are essential in many fields. Unfortunately, analyses performed using these tools can only be reproduced or adapted to other study designs if authors share their code, software version and software settings. This section examines techniques and tools for reproducible data analysis, including notebooks, version control, managers for packages, dependencies and the programming environment, and containers.

8. Data sharing: Depositing data in public repositories allows other scientists to review published results and reuse the data for additional analyses or studies. All data should adhere to the principle of FAIR data: be findable, accessible, interoperable and reusable (https://fairsharing.org/). This section describes the types of information that should be shared to allow the community to interpret and use archived data. We also discuss best practices, including criteria for selecting a repository and the importance of specifying a license for data and code reuse. There are instances where data cannot be shared, this includes when there are privacy concerns with genetic data from living people.

132

133

134

135

136

137

138

139

140

141

142

143

144

145

146

147

148

149

\section{Reproducibility for Everyone (R4E)}

R4E was formed in 2018 to address the challenges of integrating reproducible research practices in life science laboratories across the globe. Our mission is to increase awareness of the factors that affect reproducibility, and to promote best practices for reproducible and transparent scientific research. We offer open access introductory materials and workshops to teach scientists at all career stages and across disciplines about concrete steps they can take to improve the transparency and reproducibility of their research. All workshops are offered free of charge. We developed 8 modules as independent, in-depth slide sets focusing on different aspects of the day-to-day scientific workflow, allowing trainers to customize the workshop and adapt it to audiences in different disciplines (Box 1). R4E targets mainly biological and medical research practices (reagent and protocol sharing, data management) and in part computer science (bioinformatic tools) as evidenced by the range of trainings offered so far. Tools we discuss could also be useful for disciplines close to biological research like bioengineering, biophysics, (bio)chemistry, etc. Some training modules, especially Data 
management, Data visualization and Figure design, might be valuable for qualitative

All materials, including recordings of previous R4E workshops and webinars, are available at www.repro4everyone.org (RRID:SCR_018958). The goal of R4E is to provide scientists with a clear overview of existing reproducibility-promoting tools, as well as to give scientists the opportunity to revisit all training material when needed, by providing them with full access to all training materials so they learn at their own pace. In addition, we welcome each trainee to fine-tune the material for their own field of expertise and to train their peers. For trainees who want to help run one of our workshops we offer the train-the-trainer approach: We meet with the trainee before the workshop and decide together which section of the material the trainee will present. Then we go through the material together, share speaker notes and practice with the trainee if needed to stay in time during the workshop.

We have developed materials for both introductory and intensive workshop formats that are described below.

Introductory workshops are organized as two-hour sessions, including a 60- to 90minute presentation and 30-minute interactive discussion of case studies, which can be held as in-person or virtual workshops with a large number of participants (>100). These introductory workshops are designed for an interdisciplinary audience and do not require prior knowledge of reproducible research practices as they cover many different topics (Box 1). These workshops are generally presented from a team of two to four instructors.

Intensive workshops provide in-depth training in the implementation of reproducible research practices for one or more topics. These workshops take at least four hours. Depending on the number of topics covered, intensive workshops may be spread over several days. R4E members typically design these sessions to provide intensive instruction within their areas of expertise. Outside experts may also be invited to teach sessions on additional topics. This type of workshop is best suited for a smaller $(<50)$ group of participants.

\section{Box 2: Resources for training in reproducible research}

Carpentries workshops (https://carpentries.org/ ): Workshops teaching reproducible data handling and coding skills. Intended for scientists at any career stage.

Frictionless Data Fellowship (https://fellows.frictionlessdata.io ): Nine-month virtual training program on frictionless data tools and approaches. Target audience are mainly early-career researchers (ECRs). Eight fellows are selected each year and a stipend is provided.

Oxford Berlin Summer School (https://www.bihealth.org/en/notices/oxford-berlinsummer-school-on-open-research-2020 ): Five-day summer school covering open research and reproducibility in science. 
ReproducibiliTea (https://reproducibilitea.org/ ): Locally run journal clubs focused on open science and reproducibility. Target audience are mainly early career researchers. Global reach with currently 111 local groups.

Research Transparency and Reproducibility Training (RT2; https://bitss.org): Three-day training providing overview of reproducible tools and practices for social sciences. Target audience are scientists at any career stage of Social Sciences.

\section{Project TIER (Teaching Integrity in Empirical Research)}

(https://www.projecttier.org/): Training in empirical research transparency and replicability for social scientists, students and faculty. Offer fellowships and workshops for faculty and graduate students.

Framework for Open and Reproducible Research Training (FORRT;

https://forrt.org/): Connects educators and scholars in higher education to include open and reproducible science tenets in education. Offer the e-learning platform Nexus with several curated resources that include sufficient context for educators to use.

Over the years, our community has grown and diversified substantially, consisting of scientists who taught one, or many R4E workshops. To date, we have reached more than 3000 researchers through over 30 workshops, which were predominantly held at international conferences and spanned numerous life science disciplines (e.g. ecology, biotechnology, plant sciences, neuroscience and many others). In addition, we have hosted several webinars that allowed researchers from all around the world to join, including webinars for early career scientists participating in the eLife Community Ambassadors Program. Investigators and conference organizers can request to host a workshop led by our volunteers or use our materials to learn more about responsible research practices and offer their own training.

The goal of our training is to introduce participants to a reproducible scientific workflow. Individual scientists or laboratories can make their research more reproducible by implementing as many of the steps introduced in our workshops as they are comfortable with (Figure 2). Feedback on our workshops indicate that $80 \%$ of participants learned important new aspects of reproducibly research practices and are very likely to implement at least some of the presented tools in their own research workflows in future. It is important to point out that this will likely work best as a stepwise, iterative process to avoid scientists from feeling overwhelmed with implementing too many changes at once. When writing a research paper, the largest impact on the reproducibility of your work can be made by incorporating the following changes: adding a detailed list of materials used for the research, that includes research resource identifiers (RRIDs; https://scicrunch.org/resources) and catalog numbers for all materials (kits, antibodies, seeds, cell lines, organisms, etc.) that were created or used during the study. Ideally, newly generated reagents or organisms are deposited at appropriate repositories to enable easy access for other scientists. Incorporating a detailed and specific methods section is crucial to reproduce the research. Ideally, protocols are deposited at a repository, and the 
212 DOI number of the respective protocol is incorporated in the methods section. Large data sets, including all metadata, should be deposited in public data repositories to generate findable, accessible, interoperable, and reusable (FAIR) data (Sansone et al., 2019). Finally, bioinformatic analytic pipelines and scripts can easily be shared via Github, Anaconda, or computational containers such as Singularity. At a minimum, authors should list and cite all programs used, including version numbers and parameters. We would like to point out that a supportive environment is critical for these efforts to be properly adopted in a research environment. Being the first one to speak up about irreproducible research practices at your lab or institute can be challenging, or in some cases even isolating. In this case, getting involved with a local ReproducibiliTea journal club or reaching out to the initiative to start a chapter of your own can help you connect with like-minded individuals. Similarly, joining the R4E community and discussing these situations with our community members can help you find solutions to convince your peers and supervisors of the importance of incorporating reproducible research practices.

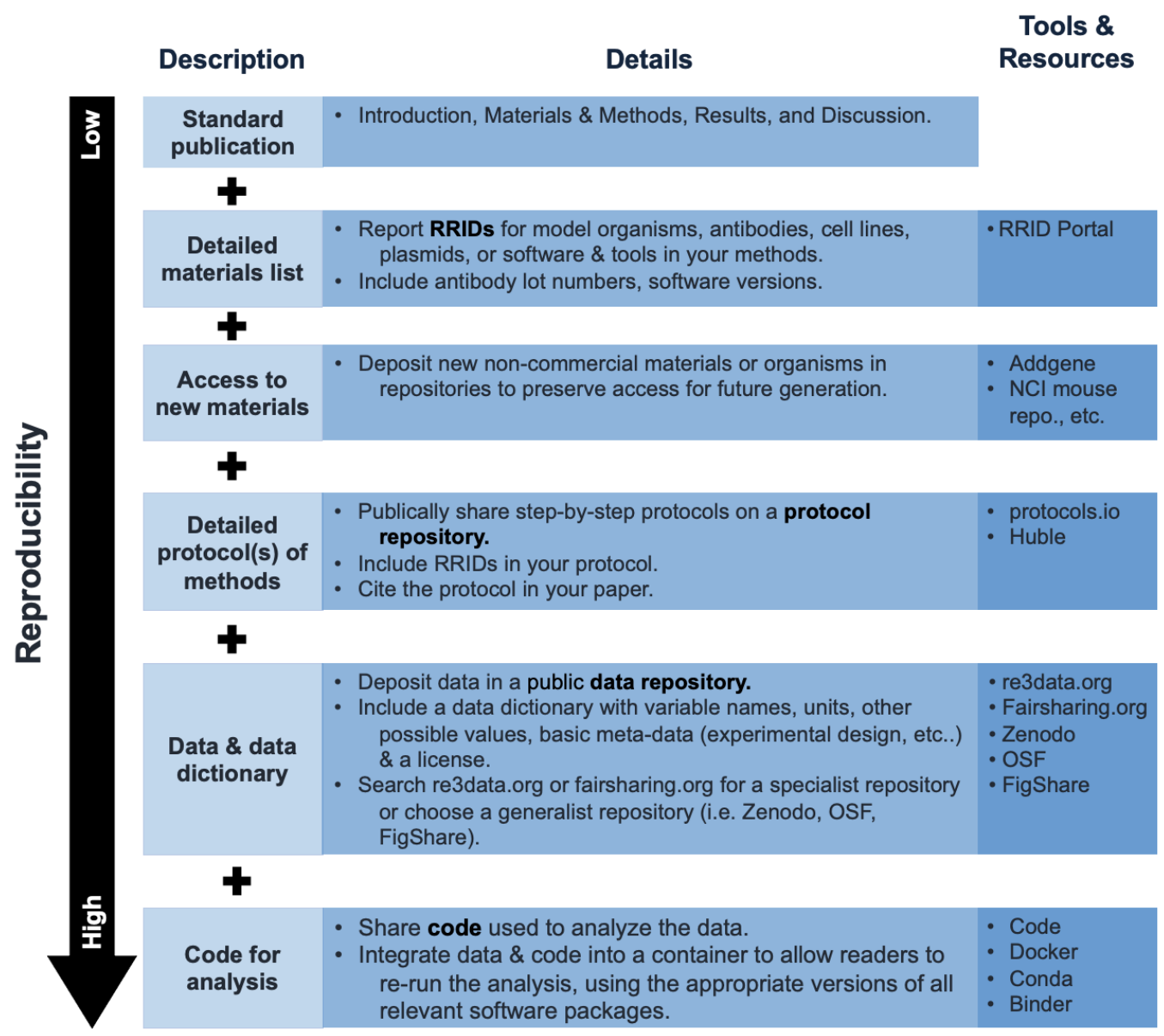

Figure 2. Standard publications often lack essential details needed to interpret or reproduce the experiment. This figure illustrates a series of items in non-descriptive random order that researchers can take one at a time or in combination to make their work easier to reproduce. Abbreviation: research resource identifiers (RRIDs).

\section{How can scientists use the R4E materials?}


the Reproducibility for Everyone team for a conference at any levels. Requests can be made via email (repro4everyone@gmail.com). Alternatively, researchers can use the 234 slides and training materials available on our website to organize their own workshops. 235 Reproducibility can be integrated into the research curriculum by asking trainees to organize and run a poster workshop at an institutional or departmental research day. Trainees can also discuss individual topics at journal clubs or as part of a methods course, after which they can develop plans to implement the identified solutions in their own research. Upcoming workshops and other opportunities to get involved and contribute will be shared through our Twitter account (@repro4everyone) and website (https://www.repro4everyone.org/).

\section{Conclusions}

Widespread adoption of new tools and practices is urgently needed to make scientific publications more transparent and reproducible. This transition will require scalable and adaptable approaches to reproducibility education that allow scientists to efficiently learn new skills and share them with others in their lab, department and field. R4E demonstrates how a common, public set of materials curated and maintained by a small group may form the basis for a global initiative to improve transparency and reproducibility in the life-sciences. Flexible materials allow instructors to adapt both the content and workshop format to meet the needs of the audience in their discipline. Continued training on reproducibility could be promoted in the laboratory by for instance changing every $\mathrm{n}^{\text {th }}$ journal club to an educational meeting, discussing the latest developments in the reproducibility field. Our workshops have reached over 3000 learners on six continents and continue to expand each year, offering a unique opportunity to train the next generation of scientists. Moving forward, R4E plans to broaden our reach by translating the existing materials into different languages and bring reproducibility training to more non-native English-speaking scientists. Increasing training in reproducible research practices alone will not suffice to make all scientific findings reproducible. To achieve this goal, higher-level changes are needed to reduce the hypercompetitive nature of scientific research. Large structural and cultural changes are needed to transition from rewarding only breakthrough scientific findings, to promoting those that were performed using reproducible and transparent research practices. 


\section{Acknowledgements}

267 Members of the Reproducibility for Everyone initiative would like to thank all 268 organizers, volunteers and staff who have helped over the years with running our 269 workshops.

270

We would like to thank the eLife Ambassador program, Addgene, Protocols.io, American Society of Plant Biology, American Society of Microbiology, and many others for

274 supporting the Reproducibility for Everyone initiative.

\section{Conflicts of Interest}

None

\section{Funding}

This work was supported by a Mozilla Mini-grant (MF-1811-05938) and the Chan Zuckerberg Initiative (Grant number: 223046). 
292

293

294

295

296

297

298

299

300

301

302

303

304

305

306

307

308

309

310

311

312

313

314

315

316

317

318

319

320

321

322

323

324

325

326

327

328

329

330

331

332

333

334

335

336

337

338

\section{References}

Alsheikh-Ali AA, Qureshi W, Al-Mallah MH, Ioannidis JPA. 2011. Public Availability of Published Research Data in High-Impact Journals. PLoS ONE 6:e24357. doi:10.1371/journal.pone.0024357

Amaral OB, Neves K, Wasilewska-Sampaio AP, Carneiro CF. 2019. The brazilian reproducibility initiative. eLife 8. doi:10.7554/eLife.41602

Baker D, Lidster K, Sottomayor A, Amor S. 2014. Two Years Later: Journals Are Not Yet Enforcing the ARRIVE Guidelines on Reporting Standards for Pre-Clinical Animal Studies. PLoS Biology 12:e1001756. doi:10.1371/journal.pbio.1001756

Baker M, Penny D. 2016. Is there a reproducibility crisis? Nature. doi:10.1038/533452A Barba LA. Terminologies for reproducible research. arXiv.org. https://arxiv.org/abs/1802.03311

Begley CG, Ellis LM. 2012. Drug development: Raise standards for preclinical cancer research. Nature 483:531-533. doi:10.1038/483531a

Botvinik-Nezer R, Holzmeister F, Camerer CF, Dreber A, Huber J, Johannesson M, Kirchler M, Iwanir R, Mumford JA, Adcock RA, Avesani P, Baczkowski BM, Bajracharya A, Bakst L, Ball S, Barilari M, Bault N, Beaton D, Beitner J, Benoit RG, Berkers RMWJ, Bhanji JP, Biswal BB, Bobadilla-Suarez S, Bortolini T, Bottenhorn KL, Bowring A, Braem S, Brooks HR, Brudner EG, Calderon CB, Camilleri JA, Castrellon JJ, Cecchetti L, Cieslik EC, Cole ZJ, Collignon O, Cox RW, Cunningham WA, Czoschke S, Dadi K, Davis CP, Luca A De, Delgado MR, Demetriou L, Dennison JB, Di X, Dickie EW, Dobryakova E, Donnat CL, Dukart J, Duncan NW, Durnez J, Eed A, Eickhoff SB, Erhart A, Fontanesi L, Fricke GM, Fu S, Galván A, Gau R, Genon S, Glatard T, Glerean E, Goeman JJ, Golowin SAE, González-García C, Gorgolewski KJ, Grady CL, Green MA, Guassi Moreira JF, Guest O, Hakimi S, Hamilton JP, Hancock R, Handjaras G, Harry BB, Hawco C, Herholz P, Herman G, Heunis S, Hoffstaedter F, Hogeveen J, Holmes S, Hu CP, Huettel SA, Hughes ME, Iacovella V, Iordan AD, Isager PM, Isik AI, Jahn A, Johnson MR, Johnstone T, Joseph MJE, Juliano AC, Kable JW, Kassinopoulos M, Koba C, Kong XZ, Koscik TR, Kucukboyaci NE, Kuhl BA, Kupek S, Laird AR, Lamm C, Langner R, Lauharatanahirun N, Lee H, Lee S, Leemans A, Leo A, Lesage E, Li F, Li MYC, Lim PC, Lintz EN, Liphardt SW, Losecaat Vermeer AB, Love BC, Mack ML, Malpica N, Marins T, Maumet C, McDonald K, McGuire JT, Melero H, Méndez Leal AS, Meyer B, Meyer KN, Mihai G, Mitsis GD, Moll J, Nielson DM, Nilsonne G, Notter MP, Olivetti E, Onicas AI, Papale P, Patil KR, Peelle JE, Pérez A, Pischedda D, Poline JB, Prystauka Y, Ray S, Reuter-Lorenz PA, Reynolds RC, Ricciardi E, Rieck JR, Rodriguez-Thompson AM, Romyn A, Salo T, Samanez-Larkin GR, Sanz-Morales E, Schlichting ML, Schultz DH, Shen Q, Sheridan MA, Silvers JA, Skagerlund K, Smith A, Smith D V., Sokol-Hessner P, Steinkamp SR, Tashjian SM, Thirion B, Thorp JN, Tinghög G, Tisdall L, Tompson SH, Toro-Serey C, Torre Tresols JJ, Tozzi L, Truong V, Turella L, van 't Veer AE, Verguts T, Vettel JM, Vijayarajah S, Vo K, Wall MB, Weeda WD, Weis S, White DJ, Wisniewski D, Xifra-Porxas A, Yearling EA, Yoon S, Yuan R, Yuen KSL, Zhang L, Zhang X, Zosky JE, Nichols TE, Poldrack RA, Schonberg T. 2020. Variability in the analysis of a single neuroimaging dataset by many teams. Nature 582:84-88. doi:10.1038/s41586-020-2314-9 
Button KS, Ioannidis JPA, Mokrysz C, Nosek BA, Flint J, Robinson ESJ, Munafò MR. 2013. Power failure: Why small sample size undermines the reliability of neuroscience. Nature Reviews Neuroscience 14:365-376. doi:10.1038/nrn3475

Collaboration OS. 2015. Estimating the reproducibility of psychological science. Science 349:aac4716-aac4716. doi:10.1126/science.aac4716

Cova F, Strickland B, Abatista A, Allard A, Andow J, Attie M, Beebe J, Berniūnas R, Boudesseul J, Colombo M, Cushman F, Diaz R, N'Djaye Nikolai van Dongen N, Dranseika V, Earp BD, Torres AG, Hannikainen I, Hernández-Conde J V., Hu W, Jaquet F, Khalifa K, Kim H, Kneer M, Knobe J, Kurthy M, Lantian A, Liao S yi, Machery E, Moerenhout T, Mott C, Phelan M, Phillips J, Rambharose N, Reuter K, Romero F, Sousa P, Sprenger J, Thalabard E, Tobia K, Viciana H, Wilkenfeld D, Zhou X. 2020. Estimating the Reproducibility of Experimental Philosophy. Review of Philosophy and Psychology 1-36. doi:10.1007/s13164-018-0400-9

Fomel S, Claerbout JF. 2009. Guest editors' introduction: Reproducible research. Computing in Science and Engineering. doi:10.1109/MCSE.2009.14

Fraser H, Parker T, Nakagawa S, Barnett A, Fidler F. 2018. Questionable research practices in ecology and evolution. PLOS ONE 13:e0200303. doi:10.1371/journal.pone. 0200303

Freedman LP, Cockburn IM, Simcoe TS. 2015. The Economics of Reproducibility in Preclinical Research. PLOS Biology 13:e1002165. doi:10.1371/journal.pbio.1002165

Friedl P. 2019. Reproducibility in cancer biology: Rethinking research into metastasis. eLife. doi:10.7554/eLife.53511

Gentleman R, Temple Lang D. 2007. Statistical analyses and reproducible research. Journal of Computational and Graphical Statistics. doi:10.1198/106186007X178663

Hardwicke TE, Mathur MB, MacDonald K, Nilsonne G, Banks GC, Kidwell MC, Mohr AH, Clayton E, Yoon EJ, Tessler MH, Lenne RL, Altman S, Long B, Frank MC. 2018. Data availability, reusability, and analytic reproducibility: Evaluating the impact of a mandatory open data policy at the journal Cognition. Royal Society Open Science 5. doi:10.1098/rsos. 180448

Head ML, Holman L, Lanfear R, Kahn AT, Jennions MD. 2015. The Extent and Consequences of P-Hacking in Science. PLOS Biology 13:e1002106. doi:10.1371/journal.pbio.1002106

Kerr NL. 1998. HARKing: Hypothesizing After the Results are Known. Personality and Social Psychology Review 2:196-217. doi:10.1207/s15327957pspr0203_4

Lazic SE. 2010. The problem of pseudoreplication in neuroscientific studies: Is it affecting your analysis? BMC Neuroscience 11:5. doi:10.1186/1471-2202-11-5

Leek JT, Peng Associate Professor of Biostatistics RD, Professor of Biostatistics A. 2015. Opinion: Reproducible research can still be wrong: Adopting a prevention approach. Proc Natl Acad Sci U S A 112:1645-6. doi:10.1073/pnas.1421412111

Lawrence, Katherine A., Michael Zentner, Nancy Wilkins-Diehr, Julie A. Wernert, Marlon Pierce, Suresh Marru, and Scott Michael. "Science gateways today and tomorrow: positive perspectives of nearly 5000 members of the research community." Concurrency and Computation: Practice and Experience 27, no. 16 (2015): 4252-4268. 
386

387

388

389

390

391

392

393

394

395

396

397

398

399

400

401

402

403

404

405

406

407

408

409

410

411

412

413

414

415

416

417

418

419

420

421

422

423

424

425

426

427

428

429

430
Mangul S, Mosqueiro T, Abdill RJ, Duong D, Mitchell K, Sarwal V, Hill B, Brito J, Littman RJ, Statz B, Lam AK-M, Dayama G, Grieneisen L, Martin LS, Flint J, Eskin E, Blekhman R. 2019. Challenges and recommendations to improve the installability and archival stability of omics computational tools. PLOS Biology 17:e3000333. doi:10.1371/journal.pbio.3000333

Marqués G, Pengo T, Sanders MA. 2020. Imaging methods are vastly underreported in biomedical research. eLife 9:1-10. doi:10.7554/ELIFE.55133

Mesirov JP. 2010. Accessible reproducible research. Science. doi:10.1126/science. 1179653

Miyakawa T. 2020. No raw data, no science: Another possible source of the reproducibility crisis. Molecular Brain. doi:10.1186/s13041-020-0552-2

Munafò MR, Nosek BA, Bishop DVM, Button KS, Chambers CD, Percie Du Sert N, Simonsohn U, Wagenmakers EJ, Ware JJ, Ioannidis JPA. 2017. A manifesto for reproducible science. Nature Human Behaviour. doi:10.1038/s41562-016-0021

Peng RD. 2011. Reproducible research in computational science. Science. doi:10.1126/science. 1213847

Prinz F, Schlange T, Asadullah K. 2011. Believe it or not: How much can we rely on published data on potential drug targets? Nature Reviews Drug Discovery. doi:10.1038/nrd3439-c1

Rigor and Reproducibility | National Institutes of Health (NIH). 2020. https://www.nih.gov/research-training/rigor-reproducibility

Sansone SA, McQuilton P, Rocca-Serra P, Gonzalez-Beltran A, Izzo M, Lister AL, Thurston M. 2019. FAIRsharing as a community approach to standards, repositories and policies. Nature Biotechnology. doi:10.1038/s41587-019-0080-8

Schloss PD. 2018. identifying and overcoming threats to reproducibility, replicability, robustness, and generalizability in microbiome research. mBio 9. doi:10.1128/mBio.00525-18

Shen K, Qi Y, Song N, Tian C, Rice SD, Gabrin MJ, Brower SL, Symmans WF, O'Shaughnessy JA, Holmes FA, Asmar L, Pusztai L. 2012. Cell line derived multigene predictor of pathologic response to neoadjuvant chemotherapy in breast cancer: A validation study on us oncology 02-103 clinical trial. BMC Medical Genomics 5:51. doi:10.1186/1755-8794-5-51

Stevens JR. 2017. Replicability and Reproducibility in Comparative Psychology. Frontiers in Psychology 8:862. doi:10.3389/fpsyg.2017.00862

Strasak AM, Zaman Q, Marinell G, Pfeiffer KP, Ulmer H. 2007. The use of statistics in medical research: A comparison of the new England journal of medicine and nature medicine. American Statistician. doi:10.1198/000313007X170242

Weissgerber TL, Milic NM, Winham SJ, Garovic VD. 2015. Beyond Bar and Line Graphs: Time for a New Data Presentation Paradigm. PLoS Biology 13:1-10. doi:10.1371/journal.pbio.1002128

Weissgerber TL, Winham SJ, Heinzen EP, Milin-Lazovic JS, Garcia-Valencia O, Bukumiric Z, Savic MD, Garovic VD, Milic NM. 2019. Reveal, Don't Conceal: Transforming Data Visualization to Improve Transparency. Circulation 140:15061518. doi:10.1161/CIRCULATIONAHA.118.037777 


\section{Technical}

- Contaminated cell lines

- Unvalidated antibodies and kits

- Natural variability

- Batch effects

\section{Human}

- Insufficient detail about methods, materials

- Poor sharing of reagents, data, and code

- Lack of version control

- Mistakes

- Publication bias

\section{Study Design \& Statistics}

- Design flaws

- Misunderstanding statistics

- HARKing

- P-hacking

- Confirmation bias

- Selective reporting

\section{External}

- Rewards for significant results, publication in high impact journals

- Lack of incentives for responsible research practices

- Paywalls

- Hyper competitiveness

- Fraud

- Sensitivity to conditions, equipment, and staff

Figure 1. Approximation of the classification of categories that contribute to irreproducible scientific results. The factors include technical, human, errors in study design and statistical analysis, and external. Specific examples have been listed under each factor that contribute to irreproducible scientific results. 


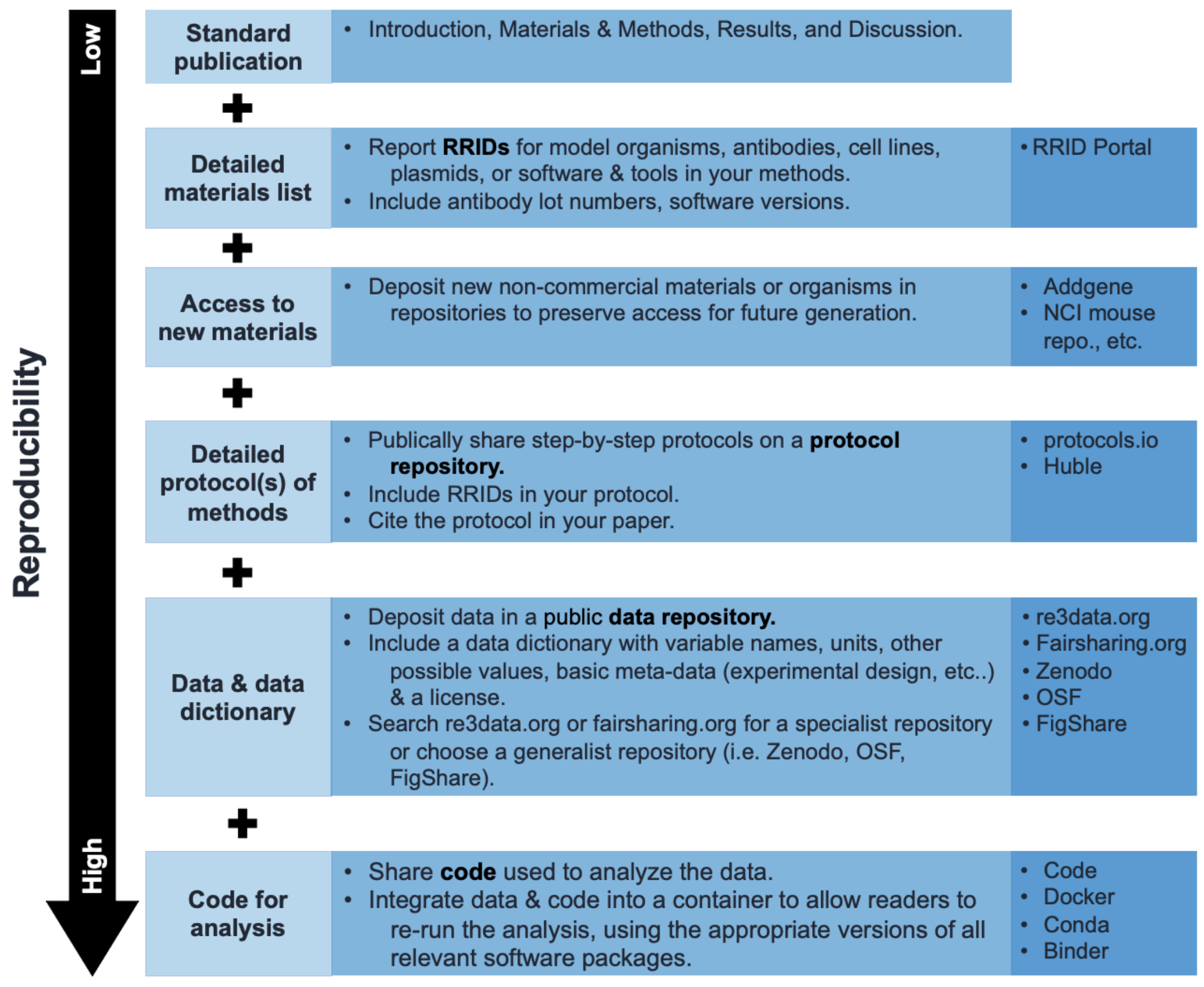

Figure 2. Standard publications often lack essential details needed to interpret or reproduce the experiment. This figure illustrates a series of items in non-descriptive random order that researchers can take one at a time or in combination to make their work easier to reproduce. Abbreviation: research resource identifiers (RRIDs). 